\title{
Pseudopneumothorax: Emphysema Case Mimicking Pneumothorax
}

\author{
(1) Leyla Öztürk Sönmez¹, (1) Togay Evrin², (1) Mustafa Kürşat Ayranci³ , (1) Erden Erol Ünlüer \\ ${ }^{1}$ Clinic of Emergency Medicine, Beyhekim State Hospital, Konya, Turkey \\ 2Department of Emergency Medicine, Ufuk University Faculty of Medicine, Ankara, Turkey \\ ${ }^{3}$ Department of Emergency Medicine, Necmettin Erbakan University Faculty of Medicine, Konya, Turkey \\ ${ }^{4}$ Department of Emergency Medicine, Ușak University Faculty of Medicine, Ușak, Turkey
}

\begin{abstract}
Bedside lung ultrasound (LUS) is generally emergency rooms for the patients suspected to have lung pathology. LUS is especially important for quick diagnosis and fast treatment, especially in unstable patients. Pneumothorax is the condition of air entering between pleural plaques and lung being separated from the thoracic wall. Typically, there is a low amount of lubricating fluid between visceral pleura covering the lung and parietal pleura covering the inner face of the thoracic wall. Emphysema is the abnormal widening of air sacs distal to the terminal bronchioles with the destruction of alveolar walls without fibrosis, and it is one of the obstructive lung diseases. Cases, where emphysema is confused with pneumothorax, were also defined rarely in the literature. We wanted to create awareness on the place of ultrasound on pneumothorax diagnosis by sharing the emphysema case imitating pneumothorax in LUS seen rather rare in literature.
\end{abstract}

Keywords: Lung ultrasound, pneumothorax, emphysema, lung air cysts

\section{Introduction}

Pneumothorax is the condition of air entering between pleural membranes that causes lung being separated from the thoracic wall. Typically, there is a low amount of lubricating fluid between visceral pleura covering the lung and parietal pleura covering the inner face of the thoracic wall. Pneumothorax is examined under three titles as primary, secondary, and tension pneumothorax. Treatment is determined according to the amount of air and pneumothorax type. Evaluation of standing inspiration and expiration chest $\mathrm{X}$-ray is primarily suggested in spontaneous pneumothorax diagnosis. The use of computed tomography (CT) is suggested in cases with uncertain diagnosis or complex cases (1).

Emphysema is the abnormal widening of air sacs distal to the terminal bronchioles with the destruction of alveolar walls without fibrosis, and it is one of the obstructive lung diseases. It has three types; centrilobular, panlobular, and paraseptal (2).

Bedside lung ultrasound (LUS) is generally performed in emergency rooms (ER) to the patients suspected to have lung pathology. LUS is especially important for quick diagnosis and fast treatment, particularly in unstable patients. LUS may provide to make a diagnosis without ionizing radiation exposure in diseases such as pleural effusion, interstitial syndromes, pneumothorax and pneumonia, and ultrasound is now seen as a way to provide speed in all kinds of time consuming works including difficult peripheric vascular access interventions to decrease complication rate in $\mathrm{ER}(3,4)$.

Cases, where emphysema is confused with pneumothorax, were also reported rarely in the literature (5). We aimed to draw attention to the application of ultrasound for the detection of pneumothorax by sharing the emphysema case mimicking pneumothorax in LUS that seen rather rare in literature.

\section{Case Report}

Fifty years old male patient referred to ER with the complaint of hemoptysis. Vital findings were as follows at referral: Noninvasive blood pressure: $107 / 69 \mathrm{mmHg}$, heart rate: 60/min, oxygen saturation $\left(\mathrm{SaO}_{2}\right): 96 \%$, body temperature: $37.1{ }^{\circ} \mathrm{C}$. 
The patient had asthma and was not using any medicine other than daily inhaled budesonide, formoterol fumarate, and tiotropium bromide. The patient had diagnosed with tuberculosis (TB) five years ago and had an inpatient treatment period in the chest clinic. He had continued anti-TB treatment for 2.5 months but had stopped taking medicine without a doctor visit.

The patient had 200-300 cc hemoptysis at $01.30 \mathrm{am}$ and was brought to the ER in an ambulance at $02.00 \mathrm{am}$. He did not have any hemoptysis in the follow-ups, until the morning. In the physical examination of the patient who has a cachectic appearance, no pathological findings were detected apart from coarse rale in the left lung upper lobe.

No significant anomalies were detected in common blood count, biochemistry, coagulation profile, and D- dimer values studied. No anomalies were detected apart from hypoxia in arterial blood gas analysis ( $\mathrm{pH}: 7.422, \mathrm{pCO}_{2}: 38.8 \mathrm{mmHg}, \mathrm{pO}_{2 \text { : }}$ $58.6 \mathrm{mmHg}$ ). No urgent pathological changes were detected in electrocardiography.

Radiological evaluation; the diaphragm was straightened on the left and located above, spread loss of density in the right lung, reticular density increase in the right lung, and reticulonodular density increase in the left lung and spread linear atelectatic bands were observed in both lungs according to the chest X-ray taken. Atelectatic areas were observed in the left apex of the lung, and blunting was observed in left costophrenic sinus (Figure 1A).

Eight zones were evaluated at the bedside LUS. No significant pathologies were detected on the right. On the left side, no pleural movements were detected in zones 1, 2, 3, 4, and a stratosphere sign was observed. Also, a standstill B line was detected in zone 1 on the left side (Figure 1B).

Despite the existence of LUS pneumothorax findings, a pneumothorax line was not detected in chest X-ray, so thorax CT of the patient was taken considering that a small pneumothorax might be present. However, pneumothorax was not observed in thorax CT too.

In thorax CT; fibrotic and cystic changes in the upper lobe in both lungs, on the right lung, paraseptal emphysema areas, more commonly in upper lobe, $6 \mathrm{~mm}$ calcified pulmonary nodule in the lower lobe of right lung and calcification in the diaphragmal face, fibrocystic changes in upper lobe apical segment (fibrosis secondary to previous TB?) and on the left lung, bronchiectases, infiltrative consolidation areas in ground glass density, spread mostly in subpleural area and thin-walled cystic lesions were reported. Linear atelectasis was reported in both lungs, shown in Figure 2.
As a result, pneumothorax was not found in our patient who had a barcode/stratosphere sign, which is accepted specific for pneumothorax. The primary diagnosis was found to be lung emphysema reported together with changes secondary to TB. Finally, the patient consulted with pulmonology and hospitalized to perform a bronchoscopy.

\section{Discussion}

Bedside ultrasonography is successfully used as a fast diagnosis tool for some traumatic or non-traumatic diseases in ER for more than ten years (3). Its success rate is high, especially in unstable patients or patients with position limits for other imaging methods. In addition to generally being traumatic,
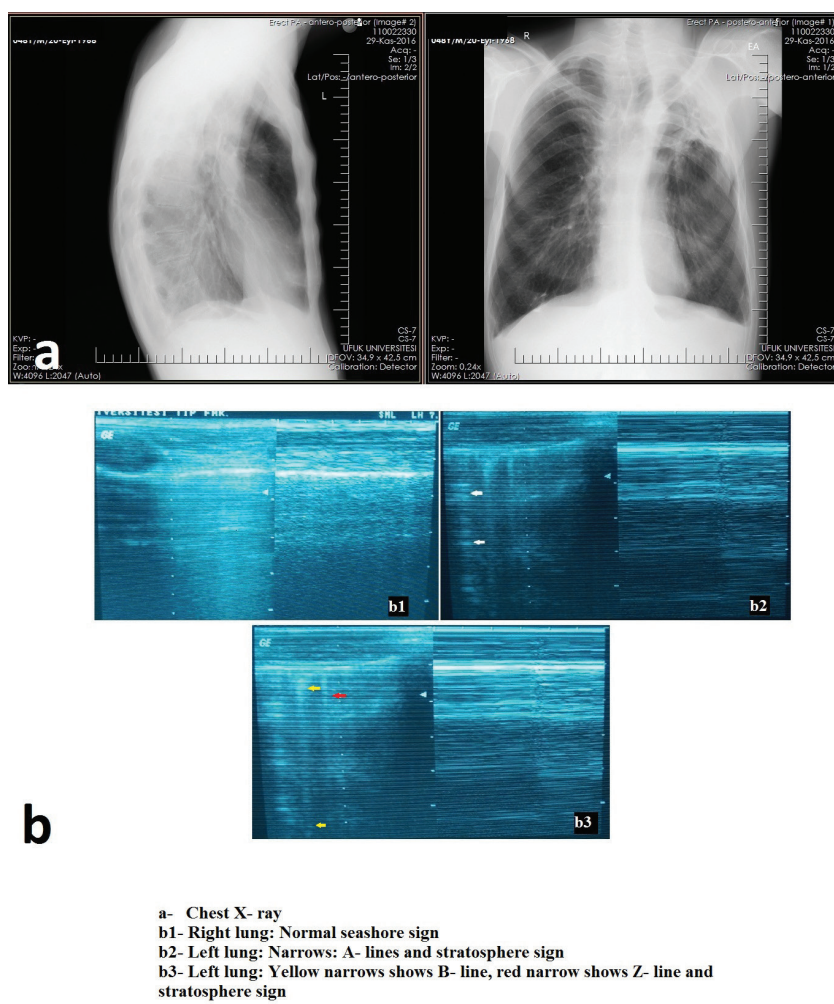

Figure 1. Images of chest $X$-ray and lung ultrasonography

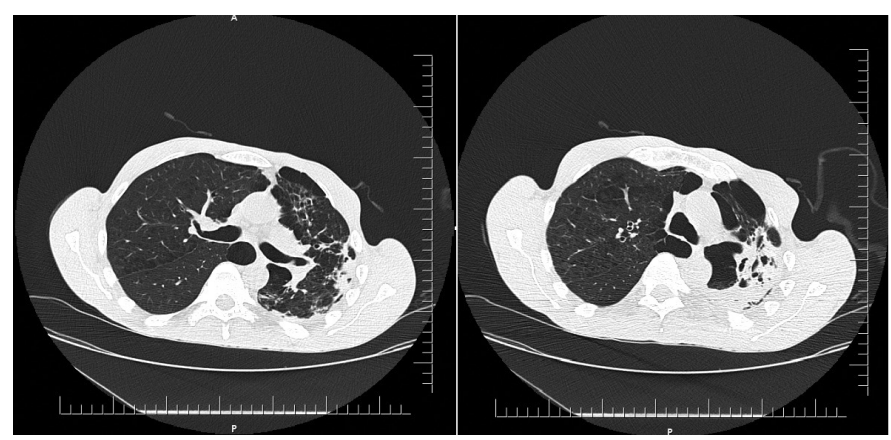

Figure 2. Images of thorax computed tomography, emphysema and air cystic structures 
pneumothorax can also be spontaneous. Traditionally, when the pneumothorax is suspected, a chest X-ray is evaluated, but its success rate was reported 36-48\% percent in different studies (6). The Eastern Association for the Surgery and Trauma renewed their guide in 2011 and gave level 2 recommendation to ultrasound in diagnosis of pneumothorax (7) Chest X-ray and LUS were compared in a retrospective review and ultrasound was found to detect pneumothorax with $93.9 \%$ sensitivity and its negative predictive value was found $99.9 \%$ (8). LUS and multidetector CT were compared in another retrospective study, and sensitivity of ultrasound in the diagnosis of pneumothorax was found $77 \%$, and specificity was found $99.9 \%$. Even though ultrasound was not found as successful as CT, there are restrictions in CT use for unstable patients (9). Additionally, in a pneumothorax case written by Unluer and Karagoz (10) a fast diagnosis was provided by showing lung point with bedside LUS in a 17 year old male patient referring to the ER with pain in left shoulder and without any story of trauma and the patient was discharged six hours later after being treated with needle aspiration. The International Liaison Committee on LUS published some proofbased suggestions for LUS in 2011 (11). In a lung with decent aeration, pleural line, lung sliding, and A-lines should be seen 2D and seashore sign should be seen in M mode so different lung pathologies can be considered ultrasonographically when these are missing (12). LUS in critically ill patients includes 10 findings such as bat sign (pleural line), lung sliding (seashore sign); A lines (horizontal artifact); quad sign showing pleural effusion (quadrant) and sinusoid sign, fractal and tissue-like sign showing consolidation in lung, B lines and lung rockets showing interstitial syndromes; stratosphere sign without observance of lung sliding causing us to consider pneumothorax and 2 other signs in addition to lung point showing pneumothorax; such as lung pulse distinguishing atelectasis from pneumonia and dynamic air bronchogram (3). Lack of or decrease in lung sliding can also be seen in complete atelectasis, massive fibrosis, abdominal compartment syndrome, severe acute asthma attack, apnea, and cardiorespiratory arrest cases (13). It should also be kept in mind that low-frequency $(2.5 \mathrm{~Hz})$ probes would not be satisfactory for detecting lung sliding movement (3).

In LUS of pneumothorax, lung sliding is not observed, A-lines are observed ahead hyperechoic parietal pleural-air border and form characteristic "stratosphere finding, barcode finding" appearance in $\mathrm{M}$ mode. The observance of the $\mathrm{B}$ line excludes pneumothorax because this line originates only from the visceral pleura-lung border. However, it should be kept in mind that Z-lines are changes that can imitate B-lines. Again the lung point, which indicates the location where the lung is separated from the chest wall, is where stratosphere finding and seashore sign are seen together in $\mathrm{M}$ mode, supports pneumothorax. As a result, four characteristic sonographic findings of pneumothorax are lack of lung sliding, B-lines and lung pulse, and the presence of lung point $(11,12,14)$.

In the case report by Kumar et al. (5), a 26-days old infant referred to ER due to respiratory distress, and pneumothorax was considered according to the chest $\mathrm{X}$-ray taken, but there was no recovery, so thorax CT was taken and finally, congenital lobar emphysema was detected (5). Emphysematous areas may imitate pneumothorax in LUS in chronic obstructive pulmonary disease (COPD) patients, according to Slater et al. (15) Thus, ultrasound may be used to exclude pneumothorax in COPD patients, but additional imaging methods are required for pneumothorax diagnosis (15).

A stratosphere sign of pneumothorax was observed in our case, but pneumothorax was not detected in the thorax $\mathrm{CT}$ taken.

\section{Conclusion}

As a result, because of the advantages of better portability, quickness, real-time imaging, cost-saving, and no exposure to ionizing radiation (especially in children and pregnant women), ultrasound is such a useful method with high applicability in critically ill patients suspected to have lung pathology. It saves time for diagnosis and provides immediate therapeutic approaches in ER, but in some complex cases, we recommend that the diagnosis should be confirmed with more advanced imaging techniques in case of clinical suspicion.

\section{Ethics}

Informed Consent: The authors declared that the research was conducted according to the principles of the World Medical Association Declaration of Helsinki "Ethical Principles for Medical Research Involving Human Subjects", (amended in October 2013). Written informed consent was obtained from patients who participated in this case.

Peer-review: Externally peer-reviewed.

\section{Authorship Contributions}

Concept: L.Ö.S., M.K.A., T.E., E.E.Ü., Design: L.Ö.S., M.K.A., T.E., E.E.Ü., Data Collection or Processing: L.Ö.S., T.E., Analysis or Interpretation: L.Ö.S., M.K.A., T.E., E.E.Ü., Literature Search: L.Ö.S., T.E., Writing: L.Ö.S., M.K.A., T.E., E.E.Ü.

Conflict of Interest: The authors declared no potential conflicts of interest concerning the research, authorship, and/or publication of this article.

Financial Disclosure: The authors received no financial support for the research, authorship, and/or publication of this article. 


\section{References}

1. MacDuff A, Arnold A, Harvey J. Management of spontaneous pneumothorax: British Thoracic Society pleural disease guideline 2010. Thorax. 2010;65(Suppl 2):ii 18- ii31.

2. Takahashi M, Fukuoka J, Nitta N, Takazakura R, Nagatani Y, Murakami Y, et al. Imaging of pulmonary emphysema: A pictorial review. Int J Chron Obstruct Pulmon Dis. 2008;3:193-204.

3. Lichtenstein D, van Hooland S, Elbers P, Malbrain ML. Ten good reasons to practice ultrasound in critical care. Anaesthesiol Intensive Ther. 2014;46:32335.

4. Aygün M, Yaman HE, Bayındır A. The use of Ultrasonography-guided peripheral intravenous access in Emergency Department Patients with Difficult Venous Access. Eurasian J Emerg Med. 2010;9:9-16.

5. Kumar S, Debata PK, Gupta R. Congenital lobar emphysema and intercostal drainage tube insertion: the common fate of an uncommon disease. J Clin Diagn Res. 2012;6:1568-70.

6. Wilkerson RG, Stone MB. Sensitivity of bedside ultrasound and supine anteroposterior chest radiographs for the identification of pneumothorax after blunt trauma. Acad Emerg Med. 2010;11-7.

7. Mowery NT, Gunter OL, Collier BR, Diaz JJ Jr, Haut E, Hildreth A, et al. Practice management guidelines for management of hemothorax and occult pneumothorax. J Trauma. 2011;70:510-8.
8. Soult MC, Weireter LJ, Britt RC, Collins JN, Novosel TJ, Reed SF, et al. Can routine trauma bay chest $\mathrm{x}$-ray be bypassed with an extended focused assessment with sonography for trauma examination? Am Surg. 2015;81:336-40.

9. Ianniello S, Di Giacomo V, Sessa B, Miele V. First-line sonographic diagnosis of pneumothorax in major trauma: accuracy of e-FAST and comparison with multidetector computed tomography. Radiol Med. 2014;119:674-80.

10. Unluer EE, Karagoz A. Bedside ultrasonographic diagnosis of pneumothorax Interv Med Appl Sci. 2014;6:133-6.

11. Volpicelli G, Elbarbary M, Blaivas M, Lichtenstein DA, Mathis G, Kirkpatrick AW. International evidence-based recommendations for point-of-care lung ultrasound. Intensive Care Med. 2012;38:577-91.

12. Saraogi A. Lung ultrasound: Present and future. Lung India. 2015;32:250-7.

13. Kristensen MS, Teoh WH, Graumann O, Laursen CB. Ultrasonography for clinical decision-making and intervention in airway management: from the mouth to the lungs and pleurae. Insights Imaging. 2014;5:253-79.

14. Lichtenstein D, Mezière G, Biderman P, Gepner A. The "lung point": An ultrasound sign specific to pneumothorax. Intensive Care Med. 2000;26:143440.

15. Slater A, Goodwin M, Anderson KE, Gleeson FV. COPD can mimic the appearance of pneumothorax on thoracic ultrasound. Chest 2006;129:545. 